\title{
ON THE GEGENSCHEIN AND THE SYMMETRY PLANE
}

\author{
S. S. HONG AND S. M. KWON \\ Department of Astronomy, Seoul National University, 151-742, KOREA
}

\begin{abstract}
Using 3-dim density models of the zodiacal cloud, we have calculated brightness of the zodiacal light over an extended region around the anti-solar point. The isophotal contours of the model Gegenscheins differ from each other, morphologically, to the degree that they can differentiate the competing density models. The recently reduced Gegenschein observations of $2^{\circ}$ resolution clearly favour the ellipsoid-type models to the fan-types, and also suggest that the surface of the densest dust concentration in the outer part of the cloud has its ascending node at longitude $100 \pm 20^{\circ}$ and is inclined $2 \pm 0^{\circ} .5$ with respect to the ecliptic plane.
\end{abstract}

\section{Introduction}

Many models of different nature have been proposed for the 3-dim distribution of the dust particles in the zodiacal cloud (see the thorough discussions by Giese et al. [1986] and references therein). According to the late Professor Giese, most models, except for the multi-lobe, surprisingly agree in that the dust density decreases by a factor of 2 within 0.2 to $0.3 \mathrm{AU}$ above the earth orbit. Yet, the morphology of the isodensity contours in the helioecliptic meridian plane looks quite different from model to model. For example, the isodensity contours of the ellipsoid-type models (ellipsoid, sombrero, cosine, ...) are of rounded shape at the ecliptic plane; while the contours of the fan-types (fan, modified-fan, extreme-fan, ...) become very peaked there. The density distribution near the ecliptic ought to be related to the particle dynamics; if so, it is of importance to differentiate the ellipsoid-types from the fan-ones.

In the previous studies, observed profiles of the zodiacal light brightness only along the helioecliptic meridian, the great circle at $90^{\circ}$ elongation, and the circles around the sun are compared with the corresponding model profiles. To distinguish the morphological characteristics of the 3dim models, observed isophotes of the zodiacal light in the $\left(\lambda-\lambda_{\odot}, \beta\right)$ plane should have been compared with their corresponding model isophotes. In order to make meaningful comparisons, however, the zodiacal light distribution has to be known over an extended region of sky with fine angular resolutions. Recently reduced (Kwon, Hong, Weinberg and Misconi 1990) observations of the zodiacal light do provide the basis for such comparisons.

\section{Isophotal Contours of the Gegenschein}

The usual brightness integral has been numerically calculated to obtain the zodiacal light brightness

A.C. Levasseur-Regourd and H. Hasegawa (eds.), Origin and Evolution of Interplanetary Dust, 147-150.

() 1991 Kluwer Academic Publishers, Printed in Japan. 


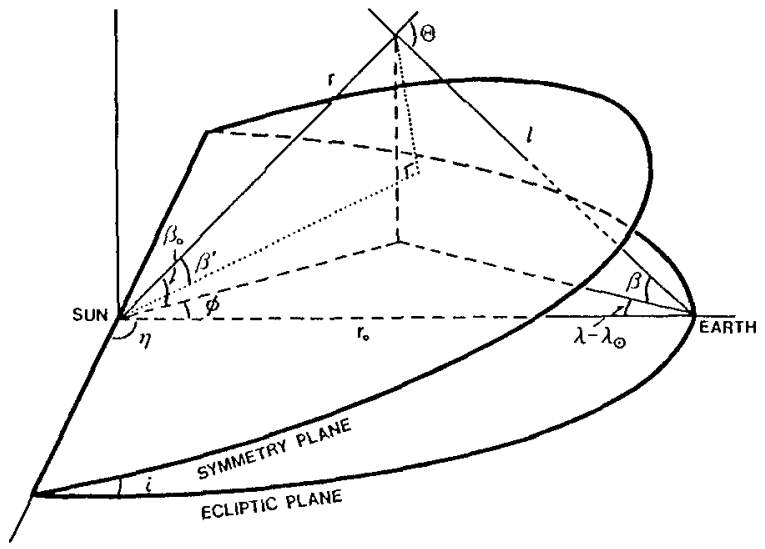

Fig 1 - Geometry involved in the brightness integral.

$Z\left(\lambda-\lambda_{\odot}, \beta\right)$ in the $\left(\lambda-\lambda_{\odot}, \beta\right)$ plane:

$$
Z\left(\lambda-\lambda_{\odot}, \beta\right)=\int_{0}^{\infty} F_{\circ}\left(\frac{r_{0}}{r}\right)^{2} n_{0}\left(\frac{r_{0}}{r}\right)^{1.3} f\left(\beta_{0}\right) \Phi(\Theta) d l .
$$

The geometry is illustrated in Fig $1 ; F_{0}$ and $n_{0}$ are the solar flux and particle number density at $r_{0}=1 \mathrm{AU}$ from the sun; and for the scattering phase function $\Phi(\Theta)$ a linear sum of three HenyeyGreenstein functions (Hong 1985) is substituted. As a representative of the ellipsoid-type models, we simply used $\left[1+\left(6.5 \sin \beta_{0}\right)^{2}\right]^{-0.65}$ (Dumont 1976) for $f\left(\beta_{0}\right)$ in the brightness integral, and that of the fan-types, $\exp \left[-2.1\left|\sin \beta_{\mathrm{o}}\right|\right]$ (Leinert et al. 1978).
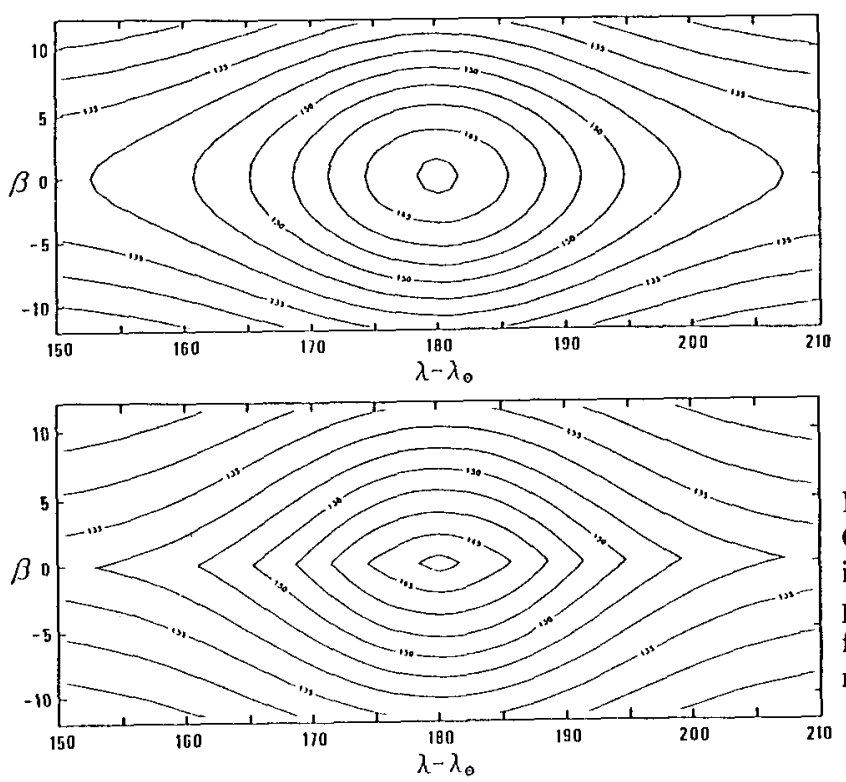

Fig 2 - Isophotes of the model Gegenscheins. The symmetry plant is assumed to be in the ecliptic plane. The ellipsoid-model is used for the upper frame, and the fanmodel for the lower one. 

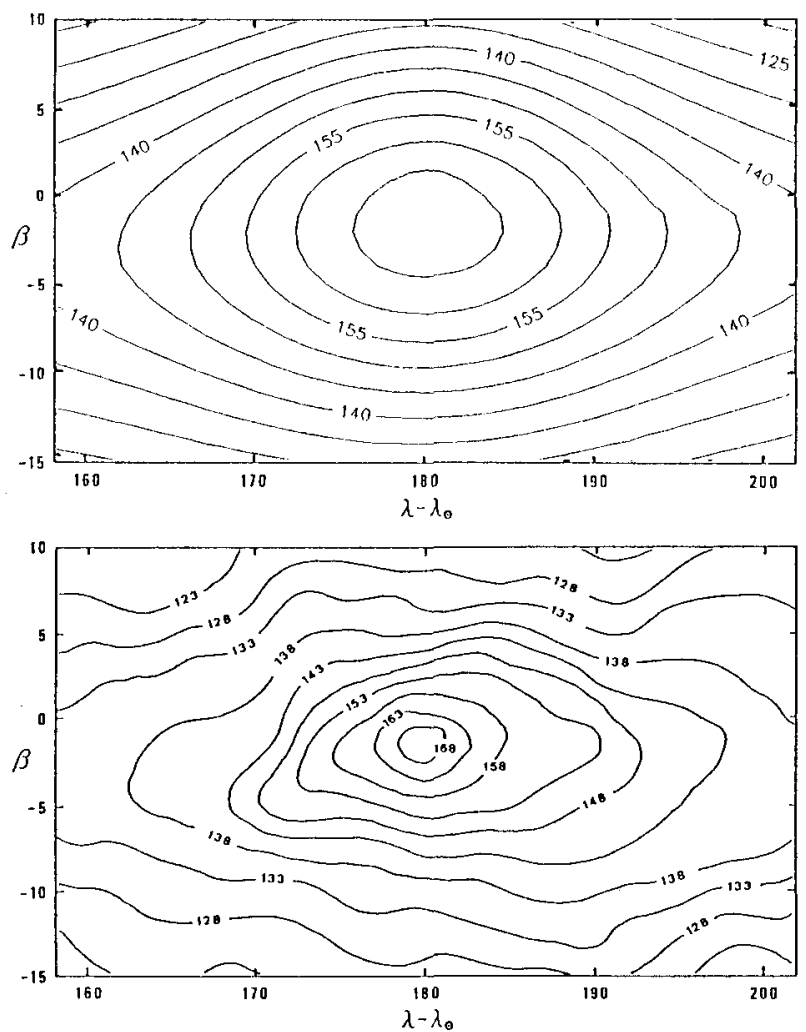

Fig 3 - Isophotes of the model Gegenschein. With the ellipsoidmodel for the 3-dim density distribution, we have substituted $i=-2^{\circ}$ and $\eta=45^{\circ}$ for the inclination and line of node of the symmetry plane.

Fig 4 - Isophotal contours of the observed Gegenschein.

The resulting isophotes are shown in the upper frame of Fig 2 for the ellipsoid-type, and in the lower frame for the fan-type. Distinct difference in the contour morphology can be noticed between the two types of cloud model. This figure have been prepared with $1^{\circ}$ resolution. If the observed resolution of Gegenschein is better than $\sim 3^{\circ}$, one could easily differentiate the cloud models on the basis of the comparison made in the figure. Previous observations of the Gegenschein (Tanabe 1965; Dumont 1965; Maucherat et al. 1986) could not quite reach this good a resolution.

To check whether the disalignment of the symmetry plane with respect to the ecliptic might smear the morphological distinctions seen in Fig 2, we have calculated the same brightness integral with $f\left(\beta_{0}\right)$ being simply replaced by $f\left(\beta^{\prime}\right)$, where $\beta^{\prime}$ is measured from the symmetry plane. As illustrated in Fig 1, the inclination of the symmetry plane is denoted by $i$, and the angle $\eta$ orients the line of nodes of the plane with respect to the sun-earth direction. The two angles, $\beta_{0}$ and $\beta^{\prime}$, are related through the relation $\sin \beta^{\prime}=\cos \beta_{0}-\sin i \cos \beta_{0} \sin (\eta+\phi)$. The distinction survives with an inclined symmetry plane.

To make a comparison with the observation, we have presented in Fig 3 the Gegenschein of the ellipsoid model with $i=-2^{\circ}$ and $\eta=45^{\circ}$. The major axis of the contour "ellipses" is slightly tilted with respect to the ecliptic, and the maximum brightness point is about $2^{\circ}$ below the anti-solar point.

The tilt angle becomes the inclination angle when $\eta=0$. When $i<0$, the isophotes above the ecliptic plane tend to bunch together; while when $i>0$, the southern lines do the same. This can also be noticed from the model calculation by Misconi (1981). The characteristics of the contour morphology outlined here hold true for the fan-type models, except that the contour shape becomes 
very peaked along the major axis.

All sky monitoring observations of night sky brightness was analyzed, with a newly developed reduction method by Kwon, Hong, Weinberg and Misconi (1990). The resulting brightness map has a resolution of $2^{\circ}$, and the Gegenschein part is reproduced in Fig 4 for a comparison. The maximum brightness point is observed at $\lambda-\lambda_{\odot} \simeq 180^{\circ}$ and $\beta \simeq-2^{\circ}$; the major axis of the elliptical isophotes is somewhat tilted with respect to the ecliptic plane; the brightness gradient is steeper in the northern side than it is in the southern side; and the closed contours are of rounded shape along the direction of their major axes.

\section{Conclusion}

The observed Gegenschein isophotes in Fig 4 agree, morphologically, with the model shown in Fig 3. Following conclusions may be drawn from the comparison: (1) The ellipsoid-type models describe the 3-dim distribution of dust in the outer part of the zodiacal cloud better than the fan-types do.

(2) The Gegenschein observations reported here are not inconsistent with the notion that the surface of the densest dust concentration has an inclination of $2 \pm 0^{\circ} .5$, and its ascending node at longitude $100 \pm 20^{\circ}$. (The data were taken, when $\lambda_{\odot}=150^{\circ}$.)

Our result on the location of symmetry plane is in agreement with those by Dumont and Sanchez (1968) ad Dumont and Levasseur-Regourd (1978), and also with the suggestion by Misconi (1980). This study has demonstrated that mappings of the zodiacal light with fine resolution can differentiate the competing models of the 3-dim density distribution. Furthermore, if one monitors the Gegenschein over, say, four seasons, he could trace the symmetry plane(s), quite accurately, at least in the outer part of the zodiacal cloud.

SSH and SMK were supported by the Basic Science Research Institute Program, Korean Ministry of Education. We are grateful to the referees for giving clarifying comments.

\section{REFERENCES}

Dumont, R. 1965, Ann. d'Astrophys., 28, 265

Dumont, R. 1976, in Lecture Notes in Physics, vol. 48, eds. H. Elsässer and H. Fechtig

(Springer-Verlag:Heidelberg), p.85

Dumont, R., and Sanchez, F. 1968, Ann. d'Astrophys., 31, 293

Dumont, R., and Levasseur-Regourd, A. C. 1978, Astr. Ap., 64, 9

Giese, R. H., Kneissel, B., and Rittich, U. 1986, Icarus, 68, 395

Hong, S. S. 1985, Astr. Ap., 146, 67

Kwon, S. M., Hong, S. S., Weinberg, J. L., and Misconi, N. Y. 1990, in this volume

Leinert, C., Hanner, M., and Pitz, E. 1978, Astr. Ap., 63, 183

Maucherat, A., Llebaria, A., and Gonin, J. C. 1986, Astr. Ap., 167, 173

Misconi, N. Y. 1980, in Solid Particles in the Solar System, eds. I. Holliday, and B. A. McIntoch (Reidel:Dordrecht), p.49

Misconi, N. Y. 1981, Icarus, 47, 265

Tanabe, H. 1965, Publ. Astron. Soc. Japan, 17, 339 